\title{
Clinicopathological and molecular characterization of SMARCA4-deficient thoracic sarcomas with comparison to potentially related entities
}

Akihiko Yoshida ${ }^{1,2}$, Eisuke Kobayashi ${ }^{2,3}$, Takashi Kubo ${ }^{4}$, Makoto Kodaira ${ }^{2,5,12}$, Toru Motoi ${ }^{6}$, Noriko Motoi $^{1}$, Kan Yonemori ${ }^{2,5}$, Yuichiro Ohe ${ }^{7}$, Shun-ichi Watanabe ${ }^{8}$, Akira Kawai ${ }^{2,3}$, Takashi Kohno $^{9}$, Hiroshi Kishimoto ${ }^{10}$, Hitoshi Ichikawa ${ }^{4,11}$ and Nobuyoshi Hiraoka ${ }^{1}$

${ }^{1}$ Department of Pathology and Clinical Laboratories, National Cancer Center Hospital, Tokyo, Japan; ${ }^{2}$ Rare Cancer Center, National Cancer Center Hospital, Tokyo, Japan; ${ }^{3}$ Department of Musculoskeletal Oncology, National Cancer Center Hospital, Tokyo, Japan; ${ }^{4}$ Division of Translational Genomics, Exploratory Oncology Research \& Clinical Trial Center, National Cancer Center, Tokyo, Japan; ${ }^{5}$ Department of Medical Oncology, National Cancer Center Hospital, Tokyo, Japan; ${ }^{6}$ Department of Pathology, Tokyo Metropolitan Cancer and Infectious Diseases Center Komagome Hospital, Tokyo, Japan; ${ }^{7}$ Department of Thoracic Oncology, National Cancer Center Hospital, Tokyo, Japan; ${ }^{8}$ Department of Thoracic Surgery, National Cancer Center Hospital, Tokyo, Japan; ${ }^{9}$ Division of Genome Biology, National Cancer Center Research Institute, Tokyo, Japan; ${ }^{10}$ Department of Pathology, Saitama Children's Medical Center, Saitama, Japan and ${ }^{11}$ Department of Clinical Genomics, National Cancer Center Research Institute, Tokyo, Japan

A growing number of studies suggest critical tumor suppressor roles of the SWI/SNF chromatin remodeling complex in a variety of human cancers. The recent discovery of SMARCA4-deficient thoracic sarcomas has added to the list of tumor groups with the SMARCA4 inactivating mutation. To better characterize these tumors and establish their nosological status, we undertook a clinicopathological and molecular analysis of 12 SMARCA4-deficient thoracic sarcomas and compared them with three potentially related disease entities. Eleven men and one woman with SMARCA4-deficient thoracic sarcomas (aged 27-82 years, median 39 years) were included in the study. Most of the patients had heavy smoking exposure and pulmonary emphysema/bullae. The primary tumors were large and involved the thoracic region in all cases and simultaneously affected the abdominal cavity in 3 cases. The patients followed a rapid course, with a median survival of 7 months. Histologically, all tumors showed diffuse sheets of mildly dyscohesive, relatively monotonous, and undifferentiated epithelioid cells with prominent nucleoli. Immunohistochemically, all tumors demonstrated a complete absence (8 cases) or diffuse severe reduction (4 cases) of SMARCA4 expression. Cytokeratin, CD34, SOX2, SALL4, and p53 were expressed in 6/12, 10/12, 10/12, 10/12, and 7/10 cases, respectively. SMARCA2 expression was deficient in $11 / 12$ cases, and none (0/8) expressed claudin-4. Targeted sequencing was performed in 5 cases and demonstrated the inactivating SMARCA4 mutation in each case and uncovered alterations in TP53 (5/5), NF1 (2/5), CDKN2A (2/5), KRAS (1/5), and KEAP1 (1/5), among others. Comparative analysis supported the distinctiveness of SMARCA4-deficient thoracic sarcomas as they were distinguishable from 13 malignant rhabdoid tumors, 15 epithelioid sarcomas, and 12 SMARCA4-deficient lung carcinomas based on clinicopathological and immunohistochemical grounds. SMARCA4-deficient thoracic sarcomas constitute a unique, highly lethal entity that requires full recognition and differentiation from other epithelioid malignancies involving the thoracic region.

Modern Pathology (2017) 30, 797-809; doi:10.1038/modpathol.2017.11; published online 3 March 2017

Correspondence: Dr A Yoshida, MD, PhD, Department of Pathology and Clinical Laboratories, National Cancer Center Hospital, 5-1-1 Tsukiji, Chuo-ku, Tokyo 104-0045, Japan.

E-mail: akyoshid@ncc.go.jp

${ }^{12}$ Current address: Kodaira Hospital, Saitama, Japan.

Received 19 November 2016; revised 27 January 2017; accepted 27 January 2017; published online 3 March 2017
The switch/sucrose-non-fermenting (SWI/SNF) complex is an evolutionarily conserved ATP-dependent chromatin remodeling complex that plays important roles in transcription, differentiation, and DNA repair. Each complex is comprised of multiple subunits that have a mutually exclusive ATPdependent catalytic unit, either SMARCA4 (BRG1) 
or SMARCA2 (BRM), core regulatory subunit such as SMARCB1 (INI1, BAF47), and other variant subunits including ARID1A (BAF250a). ${ }^{1}$ Recent studies revealed critical tumor suppressor roles of SWI/ SNF complex as $20 \%$ of human cancers harbor mutations of its subunits. ${ }^{2,3}$ For example, atypical teratoid/rhabdoid tumors and its extra-cranial analog involving the kidney or soft tissues, collectively referred hereafter as malignant rhabdoid tumors, are characterized by the consistent ( $>95 \%$ ) presence of bi-allelic inactivating mutations of the SMARCB1 gene $^{4}$ and the resultant immunohistochemical loss of SMARCB1 expression. ${ }^{5}$ A subset of SMARCB1-intact malignant rhabdoid tumors harbors SMARCA4 mutations, leading to the loss of SMARCA4 immunoexpression. ${ }^{6,7}$ Similarly, more than $90 \%$ of epithelioid sarcomas lack SMARCB1 expression, ${ }^{8}$ commonly due to the homozygous deletion of the encoding gene. ${ }^{9}$ More recently, an inactivating mutation of SMARCA4 (or rarely SMARCB1) was identified in $>95 \%$ of small cell carcinomas of the ovary, hypercalcemic type, ${ }^{10-13}$ and studies showed a high utility of SMARCA4 immunohistochemical loss in the diagnosis of this rare cancer that affects young women. ${ }^{14-16}$

The SWI/SNF complex mutations can also occur in common carcinomas in older population, and examples include $A R I D 1 A$ mutations in endometrial carcinomas and SMARCA4 mutations in lung carcinomas. $^{1,3}$ SMARCA4 mutation and/or loss of SMARCA4 immunoreactivity in lung cancers occurs in $\sim 10 \%$ of poorly differentiated adenocarcinomas, which are wild type for EGFR and $A L K$, typically affect smoking men, and are associated with worse survival. ${ }^{17-19}$ In addition, SWI/SNF complex abnormality occurs in the process of carcinoma dedifferentiation. For example, SMARCA4 or SMARCB1 deficiency occurs in $24-50 \%$ of dedifferentiated endometrial adenocarcinomas, in which SWI/SNFdeficient undifferentiated growth develops from the SWI/SNF-proficient better-differentiated endometrioid adenocarcinomas. ${ }^{20-24}$ Dedifferentiated carcinomas with SMARCA4 or SMARCB1 deficiency were also reported in the ovary, colon, and urinary tract. ${ }^{25-27}$ Abnormal DNA mismatch repair protein expression was noted in $65-75 \%$ of endometrial dedifferentiated carcinomas 20,22,23 $^{2}$ and a subset of colonic dedifferentiated carcinomas, ${ }^{27}$ suggesting a potential synergistic role of the SWI/SNF and DNA mismatch repair pathways in cancer development.

Recently, Le Loarer et al. ${ }^{28}$ described 19 patients with a high-grade thoracic neoplasm that harbored SMARCA4 inactivating mutation and loss of SMARCA4 immunoexpression. Consistent clinicopathological findings and distinctive expression profile led to the proposal of a new entity, SMARCA4-deficient thoracic sarcomas. In order to better characterize this emerging entity and to establish its nosological status, we undertook a clinicopathological and molecular analysis of our archival cases and compared them with potentially related diseases.

\section{Materials and methods}

\section{Cases}

After receiving approval from the institutional review board (2014-089), we retrieved unclassifiable round cell or epithelioid-cell sarcomas from the pathology files of the National Cancer Center Hospital, Tokyo, Japan. We also identified a few tumors that had been originally diagnosed as epithelioid sarcomas despite the absence of SMARCB1 deficiency. In addition, high-grade tumors that were originally suspected or diagnosed as undifferentiated carcinomas, not otherwise specified, that either affected the thoracic region or lacked clear information on the primary sites were retrieved. Some tumor samples that met the above search criteria were also contributed from the Komagome Hospital, Tokyo, Japan. We ultimately collected 42 tumors for which materials were available, and all were immunostained with SMARCA4 antibody. As a result, 14 tumors presented with complete loss or severe diffuse reduction of nuclear immunoreactivity of SMARCA4. Of these, 2 tumors mainly affected both the ovary and uterus without significant thoracic involvement, and they were excluded from the study cohort as they may represent either small cell carcinomas of the ovary, hypercalcemic type or dedifferentiated endometrial carcinoma. The remaining 12 tumors are the subjects of the analysis.

\section{Comparative Cohort}

For comparative purposes, we collected three different tumor entities, whose relationship with SMARCA4-deficient thoracic sarcomas is potentially controversial. They included 13 malignant rhabdoid tumors (all in pediatric patients), of which 11 lacked SMARCB1 expression and 2 were deficient for SMARCA4 expression (complete loss in 1 case and marked reduction in 1 case), and 12 epithelioid sarcomas (all in adults), all of which lacked SMARCB1 expression. In addition, we retrieved 12 lung adenocarcinomas that were deficient for SMARCA4 immunoexpression (complete loss in 9 cases and severe reduction in 3 cases), 4 of which (including 1 with reduced SMARCA4 staining) were known to harbor SMARCA4 mutations as determined by previous studies. ${ }^{18,29}$ Their clinicopathological data are provided in Supplementary Tables 1-3.

\section{Immunohistochemistry}

A paraffin section of $4-\mu \mathrm{m}$ thickness was cut from the representative block of each tumor, and routinely deparaffinized. Heat-induced antigen retrieval was performed. The primary antibodies used were cytokeratin (AE1/AE3, 1:100; Dako, Glostrup, Denmark), 
SMARCB1 (25/BAF47, 1:100; BD Biosciences, Franklin Lakes, NJ, USA), SMARCA4 (EPNCIR111A, 1:200; Epitomics, Burlingame, CA, USA), SMARCA2 (HPA029981, 1:800; Sigma-Aldrich, St. Louis, MO, USA), CD34 (QBEnd10, 1:100; Dako), SALL4 (6E3, 1:1000; Abnova Corporation, Taipei, Taiwan), and SOX2 (AB5603, 1:1000; EMD Millipore, Billerica, MA, USA). p53 (DO-7, 1:100; Dako), TTF-1 (8G7G3/1, 1:100, Dako), and claudin-4 (3E2C1, 1:500, Zymed Laboratories, San Francisco, CA, USA) antibodies were used for selected entities. Because previous studies showed a common occurrence of abnormal DNA mismatch repair protein expression in SWI/SNFdeficient dedifferentiated carcinomas, ${ }^{22,23}$ we analyzed mismatch repair protein status by a proposed 2antibody panel using MSH6 (EP49, 1:400; Dako) and PMS2 (EP51, 1:200; Dako). ${ }^{30}$ The reactions were detected using the EnVision system (Dako). Reactions that labeled at least $1 \%$ of the cells were considered positive for CD34 (membranous), SALL4 (nuclear), SOX2 (nuclear), and claudin-4 (membranous). The intensity of the staining was graded as negative, weak, moderate, or strong, and the extent was classified as negative $(<1 \%), 1+(1$ to $<10 \%), 2+(10-50 \%)$, or $3+$ $(>50 \%)$. When the intensity was heterogeneous, the most prevalent intensity was recorded. p53 was considered overexpressed (positive) when $>50 \%$ of tumor cells expressed strong nuclear expression, while the reactions falling short of this criterion were deemed negative. For SMARCB1, MSH6, and PMS2, the reactivity was considered deficient when all tumor cells showed complete absence in the background of intact positive controls (ie, non-neoplastic cells). For SMARCA4 and SMARCA2, the reactivity was considered deficient when there was complete absence or severe diffuse reduction of staining in the background of intact positive controls (ie, non-neoplastic cells). Other immunohistochemical data were retrieved from the original pathology report. The results of SALL4 and CD34 for a subset of epithelioid sarcomas and malignant rhabdoid tumors were previously reported. ${ }^{31}$

\section{Genetic Analysis}

The results of cytogenetic and molecular genetic studies documented in the medical record were reviewed. In addition, to determine the mutation status of SMARCA4 and other oncologically relevant genes, targeted next generation sequencing analysis was performed using an original cancer gene panel, NCC oncopanel v4. We examined 5 cases whose tumor tissues (2 snap frozen and 3 formalin-fixed paraffin-embedded materials) were applicable to this analysis. NCC oncopanel v4 was designed using SureDesign (Agilent Technologies, Santa Clara, CA, USA) to capture all coding exons of 114 genes and reported translocated introns of 12 genes (Supplementary Table 4). Sequencing libraries were prepared using SureSelect XT reagent (Agilent Technologies). Paired-end sequencing $(2 \times 150 \mathrm{bp})$ was performed on NextSeq sequencer (Illumina, San Diego, CA, USA). Mutations (single-nucleotide variations and short insertions and deletions), gene amplifications, and gene fusions were detected using an in-house program cisCall (Kato et al., manuscript in preparation). For elimination of single-nucleotide polymorphism, we used 1000 Genomes (http://www. 1000genomes.org), ESP6500 (http://evs.gs.washing ton.edu/EVS/), Human Genetic Variation Database (http://www.genome.med.kyoto-u.ac.jp/SnpDB), and in-house Japanese germ-line single-nucleotide polymorphism data. In addition to known oncogenic mutations, COSMIC database (http://cancer.sanger. ac.uk/cosmic/)-registered mutations and truncation mutations were considered functionally important mutations as shown in Supplementary Table 5. Other mutations were judged as variants of unknown significance or single-nucleotide polymorphisms. The assay has been used in clinical practice. ${ }^{32}$

\section{Statistical Analysis}

Overall survival, which was measured from the date of the patient's presentation to the facility where treatment was initiated, was determined using the Kaplan-Meier method. The analysis was performed using SPSS software version 20.0 (IBM Corporation, Armonk, NY, USA).

\section{Results}

\section{Clinical Findings}

The clinical findings of SMARCA4-deficient thoracic sarcomas are summarized in Table 1. The tumors occurred in 11 men and 1 woman with ages that ranged from 27 to 82 years (median, 39 years). All patients were Japanese. Smoking history was available for 10 patients, 7 of whom reported heavy $(>20$ pack $\times$ years) smoking exposure. By radiography (and subsequent autopsy examination in a few cases), 10/12 patients had bilateral pulmonary emphysema, bullae, or both, including 2 patients who reported no smoking history. None of the 11 patients tested showed hypercalcemia. Tumor serum marker status was available for 9 cases, and neuron-specific enolase, carcinoembryonic antigen, squamous cell carcinoma antigen, CA125, and human chorionic gonadotropin levels were mildly increased in 6 out of 7, 1 out of 6,1 out of 5,2 out of 5 , and 2 out of 3 cases tested, respectively. CA19.9, pro-gastrin-releasing peptide, and alpha fetoprotein were not elevated in 6, 5, and 4 cases tested, respectively. The primary tumor sites were the thoracic wall, thoracic cavity, mediastinum, or the lung in 9 cases, and they were large (Figure 1). Among these, 8 tumors involved the thoracic cavity at least focally, whereas 1 tumor (in case 6) involved the axillary soft tissue without involvement of the thoracic cavity. Case 12 presented with a $7.8 \mathrm{~cm}$ mass involving the right upper lobe of the lung and the mediastinum, 
and subsequently developed a large ovarian tumor in 4 months; because the ovarian mass was undetectable at the initial workup for staging, the tumor was considered metastasis from the primary lung cancer. In 3 cases (cases 3, 9, and 11), determining the primary tumor site was problematic. Case 3 presented with a $6.5 \mathrm{~cm}$ lung mass and 2 retroperitoneal masses measuring $11 \mathrm{~cm}$ and $8.5 \mathrm{~cm}$. Case 9 presented with a $3.7 \mathrm{~cm}$ lung mass and a $9 \mathrm{~cm}$ abdominal mass. Case 11 presented with an $8 \mathrm{~cm}$ mediastinal mass and a $9 \mathrm{~cm}$ pelvic mass. No tumor arose from the extremities or genital regions. Most patients presented with metastatic diseases. The tumors were resected for palliative purposes in 2 patients, and the remaining 10 patients received biopsy only. Chemotherapy was administered to 6 patients. The chemotherapy regimens varied, which reflected the heterogeneous diagnoses originally rendered. Overall, the tumors were resistant to chemotherapy, although transient response to adriamycin-ifosfamide treatment was reportedly achieved in 1 case. Eight patients died 1-13 months after presentation because of their diseases, while 4 patients were alive with their diseases at 0.5 and 7 months after presentation. The estimated median overall survival duration was 7 months. Autopsy was performed for 2 cases.

\section{Pathological Findings}

The gross findings were available for 4 cases, and the tumors were described as white or soft. The numbers of glass slides available for the histological analyses ranged from 1 to 39 (median, 2). The tissue types were biopsies only for 8 tumors, both biopsies and autopsy specimens for 2 tumors, and resections for 2 tumors. Histologically, all tumors showed similar histology comprising diffuse sheets of mildly dyscohesive large epithelioid cells (Figures 2a and b). The tumor cells harbored amphophilic or lightly eosinophilic cytoplasm with focal clearing in some cases and their nuclei exhibited vesicular chromatin and 1-3 prominent nucleoli (Figures 2c and d). Rhabdoid cells were present in 6 cases with their number varying from rare to focal (Figure 2d inset). Two tumors contained focal spindled component (Figure 2e). The tumor cells were relatively monotonous with at most mild nuclear pleomorphism in all but 3 cases, the latter of which focally exhibited moderate degree of pleomorphism. Mitotic figures were plentiful in all cases ranging 3-12/high power field. All tumors showed geographic necrosis. No case demonstrated clear evidence of differentiation in the form of glandular formation, papillary structure, keratinization, neuroendocrine morphology, or specific mesenchymal lineage. One exceptional finding noted in 1 case (case 11) was a prominent myxoid stroma in which tumor cells grew in a reticular manner (Figure 2f). The original pathological diagnoses were inconclusive in 9 cases, all of which were however 

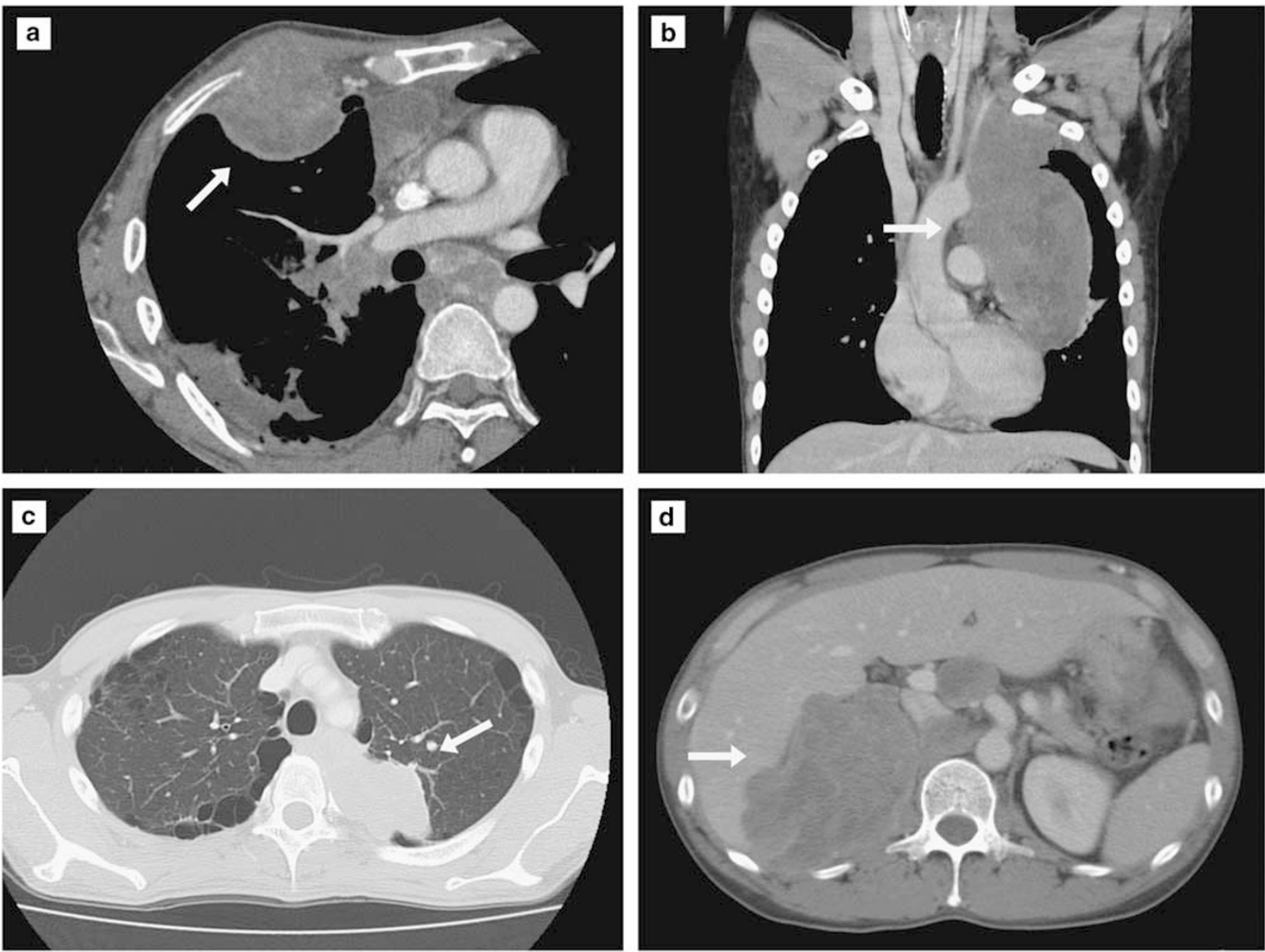

Figure 1 Computed tomography scan of SMARCA4-deficient thoracic sarcomas showed large tumors involving the thoracic structures such as the chest wall (a, case 1), the mediastinum, and/or the lung (b, case 5). In 3 cases, the patients presented with masses in both thoracic (c, case 3) and abdominal cavities (d, case 3). Most patients showed pulmonary emphysema and/or bullae (see lung parenchyma in $\mathbf{c})$. Arrows indicate masses.

interpreted as high-grade malignancy, with sarcomas or carcinomas being variably favored. Three tumors originally received definitive diagnoses of undifferentiated carcinoma, large-cell variant of Ewing sarcoma, and proximal-type epithelioid sarcoma.

\section{Immunohistochemical Findings}

The immunohistochemical results obtained for this study are summarized in Table 2. As per inclusion criteria, all tumors demonstrated complete lack (8 cases, Figure 3a) or diffuse severe reduction (4 cases, Figure 3b) of SMARCA4 expression. Cytokeratin (AE1/AE3), CD34, SOX2, and SALL4 were expressed in $6,10,10$, and 10 cases, respectively (Figures 4a-d). SMARCB1 was retained in all 9 cases tested, and SMARCA2 expression was deficient in 11 cases (Figure 4e). p53 expression was positive in 7 out of 10 cases tested, all of which exhibited diffuse strong staining in $>90 \%$ of cells
(Figure 4f). Claudin-4 was negative in all 8 cases tested. DNA mismatch repair protein status was examined for 8 tumors (cases 1-3, 6, and 9-12), and all retained PMS2 and MSH6 expression. All cases were extensively worked up at the time of original diagnosis, and the results included positive reactivity to CAM5.2 (1/1), CK7 (1/3), TTF-1 (1/10, focal), epithelial membrane antigen (8/10), vimentin (7/7), CD138 (1/2), CD99 (3/6), synaptophysin (1/4), and WT1 $(1 / 4$, nuclear). The tumors were negative for CK20 (0/3), 34ßE12 (0/1), p40 or p63 (0/5), napsin A $(0 / 3), \mathrm{HNF} 4 \alpha(0 / 5)$, NUT $(0 / 4)$, anaplastic lymphoma kinase (0/2), CD45 (0/7), CD20 (0/4), CD79a (0/4), CD3 (0/4), CD5 (0/3), TdT (0/1), CD30 (0/6), CD31 (0/4), ERG (0/2), S100 protein (0/10), HMB45 (0/2), Melan A (0/1), NKX2.2 (0/8), chromogranin A (0/3), CD56 (0/2), placental alkaline phosphatase $(0 / 3)$, c-kit (0/6), human chorionic gonadotropin $(0 / 2)$, alpha fetoprotein $(0 / 2)$, OCT3 $(0 / 7)$, MUC4 (0/2), smooth muscle actin (0/4), HHF35 $(0 / 2)$, desmin $(0 / 7)$, and myogenin $(0 / 4)$. 

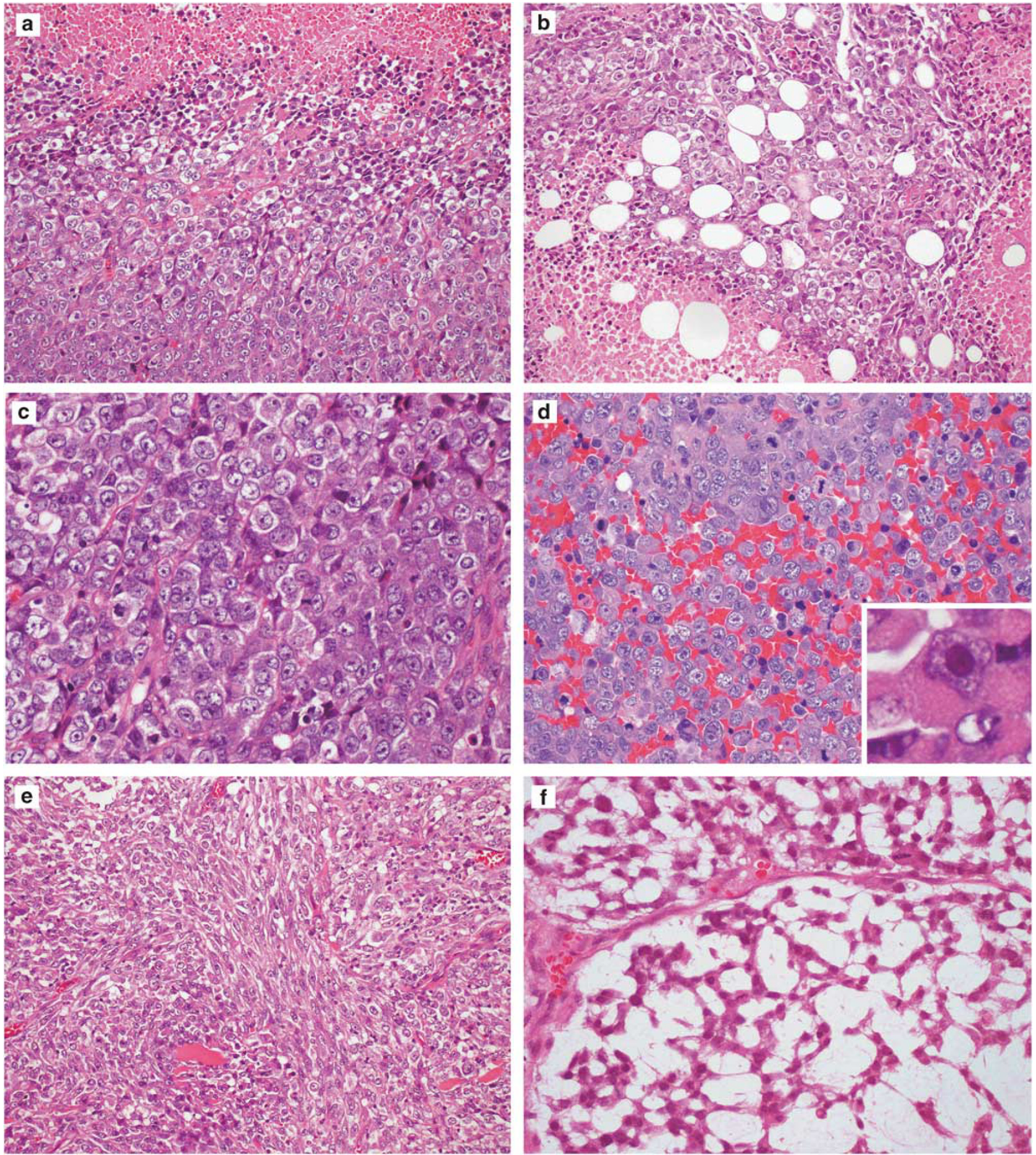

Figure 2 SMARCA4-deficient thoracic sarcomas showed diffuse sheets of large epithelioid cells (a and b). The tumor cells were relatively monotonous with mild nuclear pleomorphism and harbored amphophilic or lightly eosinophilic cytoplasm, vesicular chromatin, and prominent nucleoli (c and $\mathbf{d}$ ). Rhabdoid cells were focally present in 6 cases (d inset). Two tumors showed spindle cell component (e), and one case focally showed a prominent myxoid stroma with reticular growth (f).

\section{Molecular Findings}

Previously performed fluorescence in situ hybridization (FISH) assessments showed negative gene rearrangements for EWSR1 in 2 tumors and CIC in 2 tumors. In 3 tumors, conventional G-banded karyotyping was performed at outside institutions and all were reported to harbor complex karyotypes (see Supplementary Table 6 for details). The targeted sequencing was performed in 5 cases, each of which harbored inactivating SMARCA4 mutations, including nonsense or frame-shift mutations. Additional gene aberrations were detected in TP53, NF1, KRAS, KEAP1, CDKN2A, ARID1A, EP300, CREBBP, and 
MYC among others (Table 2 and Supplementary Table 5). The presence of $M Y C$ amplification in case 12 was verified by FISH.

\section{Clinicopathological Comparison with Potentially Related Entities}

The clinicopathological characteristics of 12 SMARCA4-deficient thoracic sarcomas were compared to those of 3 potentially related entities. The results are presented in the Supplementary Tables 1-3 and the comparative data are listed in Table 3.

Malignant rhabdoid tumors $(n=13)$ occurred in 8 boys and 5 girls with a median age of 1 year (range, 0 11 years). The primary sites were the central nervous system (atypical teratoid rhabdoid tumors) in 5 cases and extra-cranial sites in 8 cases. Malignant rhabdoid tumors demonstrated sheets of dyscohesive epithelioid to rhabdoid cells with some spindle components, a similar histomorphology to SMARCA4-deficient thoracic sarcomas. Although all SMARCA4-deficient thoracic sarcomas were deficient in SMARCA4, most (11/13) malignant rhabdoid tumors lost SMARCB1, while the remaining 2 were deficient in SMARCA4. Other immunoprofiles of malignant rhabdoid tumors were similar to SMARCA4-deficient thoracic sarcomas, in that many expressed CD34 (6/13), SOX2 (10/13), and SALL4 (12/13). In addition, similar to thoracic sarcomas, SMARCA2 expression was deficient in 6 out of 12 malignant rhabdoid tumors successfully tested. The remaining 6 cases exhibited heterogeneous SMARCA2 staining, in which geographic foci of staining loss or reduction were interspersed among immunopositive area (Supplementary Figure 1). p53 was overexpressed in none of the 13 malignant rhabdoid tumors tested.

Epithelioid sarcomas $(n=15)$ occurred in 10 men and 5 women with a median age of 34 (range, 20-52 years). They occurred in the genital/inguinal region (6), extremity (5), trunk wall (3), and head and neck (1), and histologically classified as distal (7), proximal (5), or hybrid (3) types. Although distal-type epithelioid sarcomas were morphologically distinctive due to granuloma-like arrangement, proximal-type morphology overlapped with that of SMARCA4-deficient thoracic sarcomas. Co-expression of cytokeratin and CD34 was shared by 11 out of 15 epithelioid sarcomas and 4 out of 12 SMARCA4-deficient thoracic sarcomas. However, epithelioid sarcomas were distinguished from thoracic sarcomas, because all but 1 epithelioid sarcomas lacked expression of SOX2 and SALL4, and 14 out of 15 epithelioid sarcomas retained SMARCA2 reactivity.

SMARCA4-deficient lung carcinomas $(n=12)$ occurred in 11 men and 1 woman with a median age of 55.5 years (range: 44-69 years). Most (11/12) reported a heavy smoking history. All SMARCA4deficient lung carcinomas were adenocarcinomas in this study, and morphologically different from SMARCA4-deficient thoracic sarcomas, because 

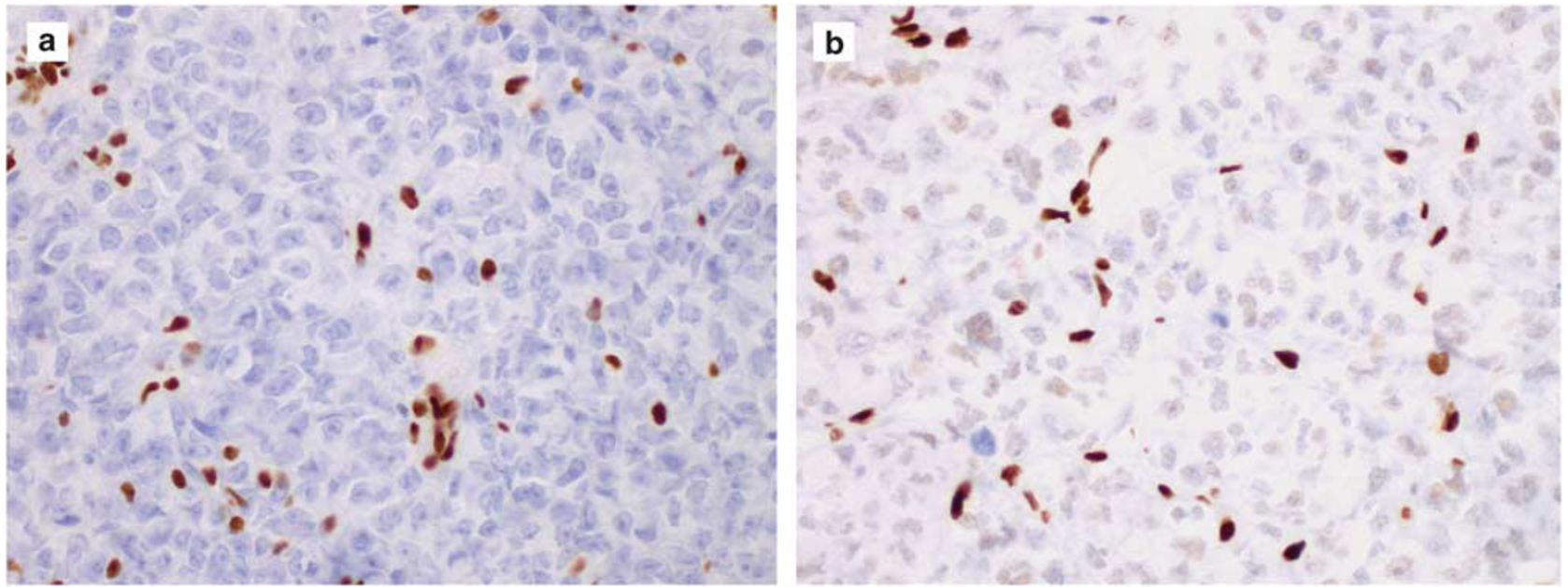

Figure 3 SMARCA4-deficient thoracic sarcomas demonstrated complete lack (8 cases, a) or severe global reduction (4 cases, b) of SMARCA4 expression. Note endothelial and inflammatory cells as internal positive controls.

carcinomas demonstrated sheets of more decidedly cohesive epithelial cells with only 1 tumor showing focal dyscohesive growth. Significant pleomorphism was present in $7 / 12$ lung carcinomas, unlike relatively uniform cytology in most thoracic sarcomas. None of the carcinomas contained rhabdoid cells. The histological growth pattern deficient in SMARCA4 expression was solid in 7 , acinar in 1 , and both solid and acinar in 4 cases. In addition, 4 carcinomas showed concomitant SMARCA4proficient better-differentiated element with a sharp border to SMARCA4-deficient growth (Supplementary Figure 2). All SMARCA4-deficient lung carcinomas expressed cytokeratin in a strong diffuse manner unlike often weak focal expression in thoracic sarcomas. TTF-1 was focally expressed in 6 of 12 lung carcinomas unlike focal weak expression in only 1 thoracic sarcoma. SOX2 expression was uncommon (4/12) in lung carcinomas with only 1 case showing diffuse strong staining, and none expressed CD34 or SALL4. SMARCA2 expression was retained in all but 1 lung carcinomas. Claudin- 4 was expressed in all 12 carcinomas.

\section{Discussion}

The clinicopathological findings of the cases reported herein are remarkably similar to those originally documented by Le Loarer et $a .^{28}$ and validate the concept of SMARCA4-deficient thoracic sarcomas. Totaling these 2 reports, 31 SMARCA4deficient thoracic sarcomas affected 27 men and 4 women (median, 40 years). Our study, however, suggested a broader disease spectrum than that originally reported. First, the clinical presentation included abdominal diseases in 3 of our cases. These tumors exhibited otherwise classic features and indeed showed simultaneous thoracic components, and in retrospect, they were compatible with thoracic primary metastatic to the abdominal cavity, although strict exclusion of abdominal primary was impossible. In this connection, our case 12 developed a large ovarian metastasis, and without clinical history, it might have been misinterpreted as small cell carcinomas of the ovary, hypercalcemic type. Along with our 2 excluded cases that involved both the uterus and ovary, these examples highlight the importance of clinical data to tumor classification.

Second, 4 of our SMARCA4-deficient thoracic sarcomas exhibited severe reduction of SMARCA4 staining rather than complete absence of reactivity. This observation expanded the data by Le Loarer et $a l,{ }^{28}$ where all the cases showed complete lack of SMARCA4 expression using the same antibody clone as in our study. The staining reduction was diffuse against the intact internal positive control, and, thus, was unlikely to be attributed to tissue degradation or fixation. ${ }^{21}$ This reduced labeling was confirmed when staining was performed at a different institution (Komagome Hosp.) for 2 selected cases (cases 3 and 10) where adequate amounts of tissue were available (data not shown). These tumors demonstrated otherwise typical features and one of them that was sequenced indeed harbored a frameshift SMARCA4 mutation, justifying their inclusion into the study cohort. Previous studies of small cell carcinomas of the ovary, hypercalcemic type documented reduced SMARCA4 expression in a small subset, some of which harbored SMARCA4 genetic inactivation. ${ }^{10,13,14}$ The relationship between SWI/ SNF gene mutation and the deficiency of its protein product is known to be complex, ${ }^{10,13,18,33-36}$ and clinicopathological findings and other marker status should be considered in problematic cases.

The nosological status of SMARCA4-deficient thoracic sarcoma can be controversial, and their relationship with malignant rhabdoid tumor, epithelioid sarcoma, and SMARCA4-deficient lung carcinoma needs particular clarification. Despite 

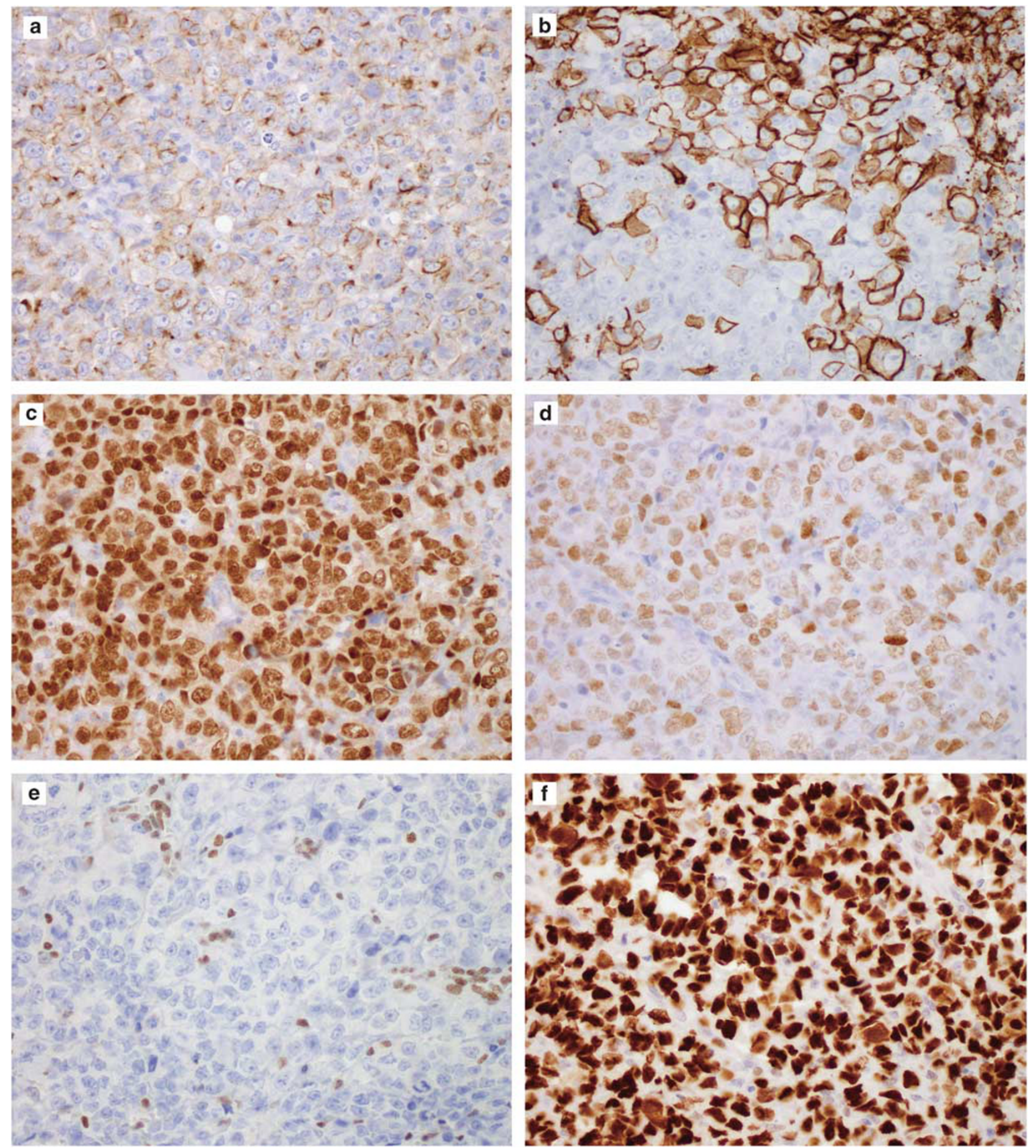

Figure 4 SMARCA4-deficient thoracic sarcomas expressed cytokeratin (a), CD34 (b), SOX2 (c), and SALL4 (d) in 50, 83, 83, and 83\% of cases, respectively. They were deficient in SMARCA2 expression in $92 \%$ of cases (e, note endothelial and inflammatory cells as internal positive controls), and p53 was overexpressed in $70 \%$ of cases (f).

overlapping morphological and expression profiles (eg, cytokeratin, CD34, SOX2, SMARCA2, and SALL4) as demonstrated in the present and previous $^{28,37,38}$ studies, malignant rhabdoid tumors affect a much younger population than SMARCA4deficient thoracic sarcomas and SMARCA4-deficient extra-cranial malignant rhabdoid tumors have thus far been documented only in young children. ${ }^{7,28}$ Malignant rhabdoid tumors are associated with germ-line mutation of SMARCB1 or SMARCA4 in a third of cases, ${ }^{7,39}$ whereas no SMARCA4 germ-line mutation has been identified in SMARCA4-deficient thoracic sarcomas. ${ }^{28}$ In contrast to a simple genomic change of malignant rhabdoid tumors ${ }^{40}$ SMARCA4- 


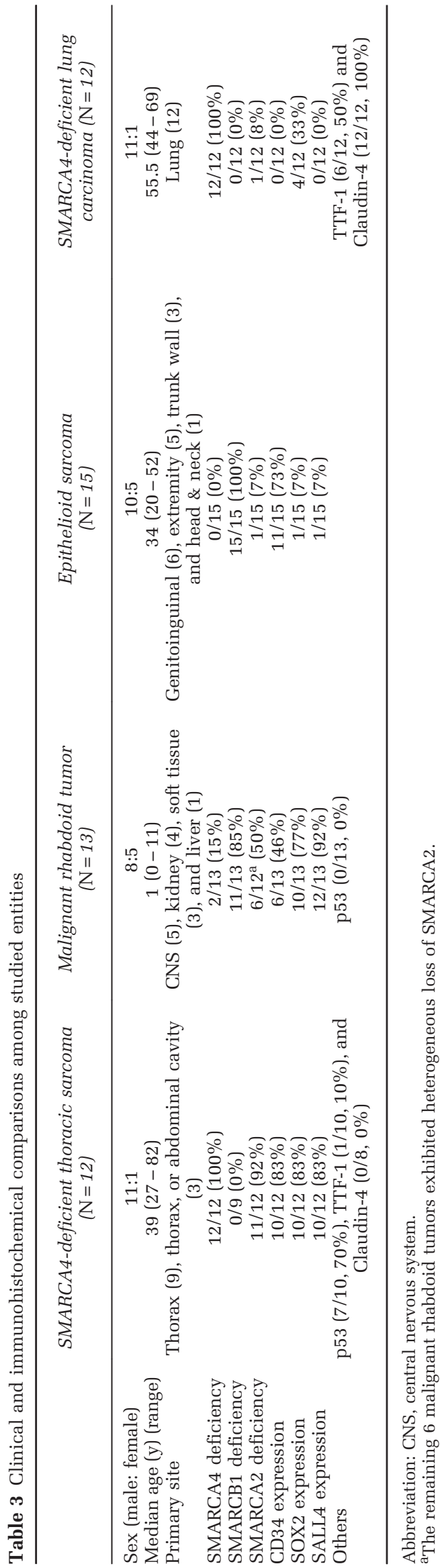

deficient thoracic sarcomas are characterized by a complex genomic profile. ${ }^{28}$ As a reflection of differential TP53 mutation status, ${ }^{28,40}$ most SMARCA4-deficient thoracic sarcomas exhibit diffuse strong p53 labeling unlike malignant rhabdoid tumors.

In contrast to epithelioid sarcomas, none of the SMARCA4-deficient thoracic sarcomas affected the extremities or genital regions. Over $90 \%$ of epithelioid sarcomas lack SMARCB1 expression and retain SMARCA4, and whether rare SMARCB1-intact epithelioid sarcomas could lack SMARCA4 instead remains unknown. Li et al. ${ }^{41}$ studied 23 epithelioid sarcomas, including 5 SMARCB1-intact examples, and found no case deficient in SMARCA4. In Le Loarer et $a l^{28} 6$ out of 18 SMARCB1-intact epithelioid sarcomas were SMARCA4 deficient on initial screening, all of which were reclassified later as SMARCA4-deficient thoracic sarcomas. SMARCA4-deficient thoracic sarcomas often express SOX2, SALL4, and typically lack SMARCA2 expression, an immunoprofile opposite to that of epithelioid sarcomas. Our case 6 was therefore exceptional, because it retained SMARCA2 and lacked SOX2 and SALL4 expression. This case involving the axillary thoracic wall was the only in our cohort for whom the tumor burden did not affect the thoracic cavity and histologically contained significant spindle cell component. This case was originally diagnosed as proximal-type epithelioid sarcoma, and it might actually represent one with novel SMARCA4 inactivating mutation in light of these phenotypic differences, although its clinical course was similar to the other cases.

Unlike SMARCA4-deficient thoracic sarcomas, most SMARCA4-deficient lung carcinomas showed cohesive solid sheets of epithelial cells with enhanced pleomorphism in some cases, a half of which also contained more differentiated growth pattern. The differential immunoprofile included staining pattern of cytokeratin (diffuse vs focal), TTF-1, CD34, SOX2, SALL4, SMARCA2, and claudin-4. Low level of SOX2 expression in our carcinoma cohort is likely related to adenocarcinoma lineage, because SOX2 is often expressed in squamous cell carcinomas. ${ }^{42}$ SALL4 can be expressed in rare $(<0.5 \%)$ lung adenocarcinomas often with fetal-lung-like morphology, ${ }^{43}$ but such a morphology was not included in our present cohort. Our study expanded the recently proposed utility of claudin-4 staining to differentiate SWI/SNF-deficient carcinomas from sarcomas. ${ }^{44}$

Although the present data highlighted the phenotypic distinctiveness of SMARCA4-deficient thoracic sarcomas as compared with lung carcinomas, a hypothesis may still be raised that they may be of epithelial derivation, in light of the consistent thoracic location, epithelioid appearance, and focal cytokeratin expression in half of the cases. These lesions have been considered sarcomas principally because of the young age of the affected patients, 
extremely large size, phenotypic similarity to malignant rhabdoid tumor, and the lack of true epithelial structures (eg, glands or papillae). However, many tumors at least focally involved the lung and the most were diagnosed by biopsy alone without examining the entire tumors. They occurred characteristically in male smokers with emphysema/ bullae and often genetically harbored C:G/A:T transversions similar to smoking-associated lung carcinomas. ${ }^{28}$ Further, among the aberrations that we identified, that involving KRAS is more commonly associated with epithelial tumors, and KEAP1 has been listed as one of the most significantly mutated gene in lung adenocarcinomas as compared to other tumor types. ${ }^{42}$ In lung carcinomas, the mutations in NF1, KRAS, and SMARCA4 are known to be more common in smoking-related adenocarcinomas. ${ }^{18,45}$ Together, these data might implicate, albeit only circumstantially, some relatedness between SMARCA4-deficient thoracic sarcomas and smoking-related lung adenocarcinomas. Rare TTF-1 expression in the former as reported previously ${ }^{28}$ and by ourselves might also be understood along this line.

In this context, the analogy of SMARCA4-deficient thoracic sarcomas to the undifferentiated component of dedifferentiated endometrial carcinomas might be worth exploring. SMARCA4-deficient dedifferentiated endometrioid carcinomas are consistently co-deficient in SMARCA2, ${ }^{22}$ and may express SALL4 and/or CD34. ${ }^{21,46}$ One example in our archive lacked claudin-4 expression (Yoshida, unpublished data). In most reported cases, the undifferentiated component predominated and sometimes eclipsed the preexisting glandular component. ${ }^{21-23}$ Similar SWI/SNF-deficient dedifferentiation and undifferentiated proliferations unaccompanied by better-differentiated element were also documented in other organs. ${ }^{25-27,44,47,48 \text {, Whether }}$ SMARCA4-deficient thoracic sarcomas might have originated via similar dedifferentiation mechanism from precursor epithelial lesions requires study involving a large number of cases, which should include rigorously dissected surgical or autopsy specimens. In contrast to some SWI/SNF-deficient dedifferentiated carcinomas, ${ }^{20,22,23}$ none of our 8 SMARCA4-deficient thoracic sarcomas tested showed abnormal DNA mismatch repair protein expression.

Differential diagnostic considerations of SMARCA4-deficient thoracic sarcomas also include germ-cell tumors, NUT-midline carcinomas, Ewing sarcomas, lymphomas, malignant mesothelioma, and thymic carcinomas, among others. Unlike diffuse strong expression in many germ-cell tumors, ${ }^{49}$ SALL4 expression in SMARCAA4-deficient thoracic sarcomas is often focal and they lack OCT3, CD30, and placental alkaline phosphatase expression. ${ }^{28}$ NUT-midline carcinomas can co-express cytokeratin and CD34, but they may show squamous differentiation and express NUT and SMARCA4. ${ }^{28}$ The CD99 staining in SMARCA4-deficient thoracic sarcomas was always focal and the more specific Ewing sarcoma marker, NKX2.2, ${ }^{50}$ was uniformly negative. Ewing sarcomas and Ewing-like sarcomas such as CIC-rearranged sarcomas retain SMARCA4 expression. ${ }^{14,15,28}$ Other diagnostic markers (eg, CD45, CD30, WT1, calretinin, p40, and c-kit) should also be helpful, although SMARCA4-deficient thoracic sarcomas may rarely express WT1 (as in case 9), $\mathrm{c}$-kit, ${ }^{28}$ or $\mathrm{p} 40 .{ }^{28}$ In addition, rare mesotheliomas were reported to lack SMARCA4 reactivity, in which case SMARCA2 expression was retained. ${ }^{28}$

In conclusion, SMARCA4-deficient thoracic sarcoma is a distinct group of high-grade malignancy that often affects young adults with smoking history and emphysematous or bullous lungs. These tumors typically present as a large thoracic mass with a rapid fatal course, but the clinical picture may be predominated by abdominal metastases. The tumors demonstrate a consistent and recognizable histology and immunophenotype, which are distinguishable in combination from malignant rhabdoid tumor, epithelioid sarcoma, and lung carcinomas. Careful clinicopathological assessment including an immunohistochemical panel (eg, SMARCA4, SMARCA2, cytokeratin, CD34, SALL4, TTF-1, and claudin-4) should solve the diagnostic dilemma for most instances including needle biopsy settings. Genetic analysis of SMARCA4-deficient thoracic sarcomas suggests some relatedness to smoking-related lung adenocarcinomas, despite phenotypic difference, requiring further study to better understand their pathogenesis.

\section{Acknowledgments}

We thank Drs Naofumi Asano, Koichi Ogura, Reiko Watanabe, Hiroshi Yoshida, Eisho Sato, Tomoyasu Kato, Naohiro Makise, Motonobu Saito, and Koji Tsuta for valuable support. They also appreciate Sachiko Miura, Chizu Kina, and Toshiko Sakaguchi for superb technical assistance. This work was supported in part by AMED (16Ack0106168h0002), the National Cancer Center Research and Development Fund (26-A-9), and JSPS Grant-in-Aid for Young Scientists (15K19065).

\section{Disclosure/conflict of interest}

The authors declare no conflict of interest.

\section{References}

1 Oike $\mathrm{T}$, Ogiwara $\mathrm{H}$, Nakano $\mathrm{T}$, et al. Inactivating mutations in SWI/SNF chromatin remodeling genes in human cancer. Jpn J Clin Oncol 2013;43:849-855.

2 Kadoch C, Hargreaves DC, Hodges C, et al. Proteomic and bioinformatic analysis of mammalian SWI/SNF 
complexes identifies extensive roles in human malignancy. Nat Genet 2013;45:592-601.

3 Shain AH, Pollack JR. The spectrum of SWI/SNF mutations, ubiquitous in human cancers. PLoS One 2013;8:e55119.

4 Jackson EM, Sievert AJ, Gai X, et al. Genomic analysis using high-density single nucleotide polymorphismbased oligonucleotide arrays and multiplex ligationdependent probe amplification provides a comprehensive analysis of INI1/SMARCB1 in malignant rhabdoid tumors. Clin Cancer Res 2009;15:1923-1930.

5 Hoot AC, Russo P, Judkins AR, et al. Immunohistochemical analysis of hSNF5/INI1 distinguishes renal and extra-renal malignant rhabdoid tumors from other pediatric soft tissue tumors. Am J Surg Pathol 2004;28: 1485-1491.

6 Hasselblatt M, Gesk S, Oyen F, et al. Nonsense mutation and inactivation of SMARCA4 (BRG1) in an atypical teratoid/rhabdoid tumor showing retained SMARCB1 (INI1) expression. Am J Surg Pathol 2011;35: 933-935.

7 Schneppenheim R, Fruhwald MC, Gesk S, et al. Germline nonsense mutation and somatic inactivation of SMARCA4/BRG1 in a family with rhabdoid tumor predisposition syndrome. Am J Hum Genet 2010;86: 279-284.

8 Hornick JL, Dal Cin P, Fletcher CD. Loss of INI1 expression is characteristic of both conventional and proximal-type epithelioid sarcoma. Am J Surg Pathol 2009;33:542-550.

9 Le Loarer F, Zhang L, Fletcher CD, et al. Consistent SMARCB1 homozygous deletions in epithelioid sarcoma and in a subset of myoepithelial carcinomas can be reliably detected by FISH in archival material. Genes Chromosomes Cancer 2014;53:475-486.

10 Jelinic P, Mueller JJ, Olvera N, et al. Recurrent SMARCA4 mutations in small cell carcinoma of the ovary. Nat Genet 2014;46:424-426.

11 Kupryjanczyk J, Dansonka-Mieszkowska A, MoesSosnowska J, et al. Ovarian small cell carcinoma of hypercalcemic type - evidence of germline origin and SMARCA4 gene inactivation. a pilot study. Pol J Pathol 2013;64:238-246.

12 Ramos P, Karnezis AN, Craig DW, et al. Small cell carcinoma of the ovary, hypercalcemic type, displays frequent inactivating germline and somatic mutations in SMARCA4. Nat Genet 2014;46:427-429.

13 Witkowski L, Carrot-Zhang J, Albrecht S, et al. Germline and somatic SMARCA4 mutations characterize small cell carcinoma of the ovary, hypercalcemic type. Nat Genet 2014;46:438-443.

14 Karanian-Philippe $\mathrm{M}$, Velasco $\mathrm{V}$, Longy $\mathrm{M}$, et al. SMARCA4 (BRG1) loss of expression is a useful marker for the diagnosis of ovarian small cell carcinoma of the hypercalcemic type (ovarian rhabdoid tumor): a comprehensive analysis of 116 rare gynecologic tumors, 9 soft tissue tumors, and 9 melanomas. Am J Surg Pathol 2015;39:1197-1205.

15 Clarke BA, Witkowski L, Ton $\mathrm{Nu} \mathrm{TN}$, et al. Loss of SMARCA4 (BRG1) protein expression as determined by immunohistochemistry in small-cell carcinoma of the ovary, hypercalcaemic type distinguishes these tumours from their mimics. Histopathology 2016;69: 727-738.

16 Conlon N, Silva A, Guerra E, et al. Loss of SMARCA4 expression is both sensitive and specific for the diagnosis of small cell carcinoma of ovary, hypercalcemic type. Am J Surg Pathol 2016;40: 395-403.

17 Matsubara D, Kishaba Y, Ishikawa S, et al. Lung cancer with loss of BRG1/BRM, shows epithelial mesenchymal transition phenotype and distinct histologic and genetic features. Cancer Sci 2013;104:266-273.

18 Oike T, Ogiwara H, Tominaga $\mathrm{Y}$, et al. A synthetic lethality-based strategy to treat cancers harboring a genetic deficiency in the chromatin remodeling factor BRG1. Cancer Res 2013;73:5508-5518.

19 Yoshimoto T, Matsubara D, Nakano T, et al. Frequent loss of the expression of multiple subunits of the SWI/SNF complex in large cell carcinoma and pleomorphic carcinoma of the lung. Pathol Int 2015;65: 595-602.

20 Hoang LN, Lee YS, Karnezis AN, et al. Immunophenotypic features of dedifferentiated endometrial carcinoma - insights from BRG1/INI1-deficient tumours. Histopathology 2016;69:560-569.

21 Strehl JD, Wachter DL, Fiedler J, et al. Pattern of SMARCB1 (INI1) and SMARCA4 (BRG1) in poorly differentiated endometrioid adenocarcinoma of the uterus: analysis of a series with emphasis on a novel SMARCA4-deficient dedifferentiated rhabdoid variant. Ann Diagn Pathol 2015;19:198-202.

22 Karnezis AN, Hoang LN, Coatham M, et al. Loss of switch/sucrose non-fermenting complex protein expression is associated with dedifferentiation in endometrial carcinomas. Mod Pathol 2016;29:302-314.

23 Stewart CJ, Crook ML. SWI/SNF complex deficiency and mismatch repair protein expression in undifferentiated and dedifferentiated endometrial carcinoma. Pathology 2015;47:439-445.

24 Donner LR, Wainwright LM, Zhang F, et al. Mutation of the INI1 gene in composite rhabdoid tumor of the endometrium. Hum Pathol 2007;38:935-939.

25 Tan A, Mohan GR, Stewart CJ. BRG1-deficient dedifferentiated endometrioid adenocarcinoma of the ovary. Pathology 2016;48:82-83.

26 Agaimy A, Bertz S, Cheng L, et al. Loss of expression of the SWI/SNF complex is a frequent event in undifferentiated/dedifferentiated urothelial carcinoma of the urinary tract. Virchows Arch 2016;469:321-330.

27 Pancione M, Remo A, Sabatino L, et al. Right-sided rhabdoid colorectal tumors might be related to the serrated pathway. Diagn Pathol 2013;8:31.

28 Le Loarer F, Watson S, Pierron G, et al. SMARCA4 inactivation defines a group of undifferentiated thoracic malignancies transcriptionally related to BAFdeficient sarcomas. Nat Genet 2015;47:1200-1205.

29 Saito M, Shimada Y, Shiraishi K, et al. Development of lung adenocarcinomas with exclusive dependence on oncogene fusions. Cancer Res 2015;75:2264-2271.

30 Shia J, Tang LH, Vakiani E, et al. Immunohistochemistry as first-line screening for detecting colorectal cancer patients at risk for hereditary nonpolyposis colorectal cancer syndrome: a 2-antibody panel may be as predictive as a 4-antibody panel. Am J Surg Pathol 2009;33:1639-1645.

31 Yoshida A, Asano N, Kawai A, et al. Differential SALL4 immunoexpression in malignant rhabdoid tumours and epithelioid sarcomas. Histopathology 2015;66:252-261.

32 Tanabe Y, Ichikawa H, Kohno T, et al. Comprehensive screening of target molecules by next-generation sequencing in patients with malignant solid tumors: guiding entry into phase I clinical trials. Mol Cancer 2016;15:73. 
33 Masliah-Planchon J, Machet MC, Freneaux P, et al. SMARCA4-mutated atypical teratoid/rhabdoid tumor with retained BRG1 expression. Pediatr Blood Cancer 2016;63:568-569.

34 Coira IF, Rufino-Palomares EE, Romero OA, et al. Expression inactivation of SMARCA4 by microRNAs in lung tumors. Hum Mol Genet 2015;24:1400-1409.

35 Kohashi K, Yamamoto H, Kumagai R, et al. Differential microRNA expression profiles between malignant rhabdoid tumor and epithelioid sarcoma: miR193a-5p is suggested to downregulate SMARCB1 mRNA expression. Mod Pathol 2014;27:832-839.

36 Tsai CY, Wong TT, Lee YH, et al. Intact INI1 gene region with paradoxical loss of protein expression in AT/RT: implications for a possible novel mechanism associated with absence of INI1 protein immunoreactivity. Am J Surg Pathol 2012;36: 128-133.

37 Kahali B, Yu J, Marquez SB, et al. The silencing of the SWI/SNF subunit and anticancer gene BRM in Rhabdoid tumors. Oncotarget 2014;5:3316-3332.

38 Rao Q, Xia QY, Wang ZY, et al. Frequent coinactivation of the SWI/SNF subunits SMARCB1, SMARCA2 and PBRM1 in malignant rhabdoid tumours. Histopathology 2015;67:121-129.

39 Hasselblatt M, Nagel I, Oyen F, et al. SMARCA4mutated atypical teratoid/rhabdoid tumors are associated with inherited germline alterations and poor prognosis. Acta Neuropathol 2014;128:453-456.

40 Fahiminiya S, Witkowski L, Nadaf J, et al. Molecular analyses reveal close similarities between small cell carcinoma of the ovary, hypercalcemic type and atypical teratoid/rhabdoid tumor. Oncotarget 2016;7: 1732-1740.

41 Li L, Fan XS, Xia QY, et al. Concurrent loss of INI1, PBRM1, and BRM expression in epithelioid sarcoma: implications for the cocontributions of multiple
SWI/SNF complex members to pathogenesis. Hum Pathol 2014;45:2247-2254.

42 Campbell JD, Alexandrov A, Kim J, et al. Distinct patterns of somatic genome alterations in lung adenocarcinomas and squamous cell carcinomas. Nat Genet 2016;48:607-616.

43 Fujimoto M, Sumiyoshi S, Yoshizawa A, et al. SALL4 immunohistochemistry in non-small-cell lung carcinomas. Histopathology 2014;64:309-311.

44 Schaefer IM, Agaimy A, Fletcher CD, et al. Claudin-4 expression distinguishes SWI/SNF complex-deficient undifferentiated carcinomas from sarcomas. Mod Pathol; advance online publication, 13 January 2017 [e-pub ahead of print].

45 Redig AJ, Capelletti M, Dahlberg SE, et al. Clinical and molecular characteristics of NF1-mutant lung cancer. Clin Cancer Res 2016;22:3148-3156.

46 Shah VI, Ramalingam P, McCluggage WG. CD34 expression in undifferentiated endometrial carcinoma. Histopathology 2016;69:894-897.

47 Agaimy A, Daum O, Markl B, et al. SWI/SNF complexdeficient undifferentiated/rhabdoid carcinomas of the gastrointestinal tract: a series of 13 cases highlighting mutually exclusive loss of SMARCA4 and SMARCA2 and frequent co-inactivation of SMARCB1 and SMARCA2. Am J Surg Pathol 2016;40:544-553.

48 Agaimy A, Haller F, Frohnauer J, et al. Pancreatic undifferentiated rhabdoid carcinoma: KRAS alterations and SMARCB1 expression status define two subtypes. Mod Pathol 2015;28:248-260.

49 Liu A, Cheng L, Du J, et al. Diagnostic utility of novel stem cell markers SALL4, OCT4, NANOG, SOX2, UTF1, and TCL1 in primary mediastinal germ cell tumors. Am J Surg Pathol 2010;34:697-706.

50 Yoshida A, Sekine S, Tsuta K, et al. NKX2.2 is a useful immunohistochemical marker for Ewing sarcoma. Am J Surg Pathol 2012;36:993-999.

Supplementary Information accompanies the paper on Modern Pathology website (http://www.nature.com/ modpathol). 